\title{
Gravidez na adolescência: um olhar sobre um fenômeno complexo
}

\author{
Ana Cristina Garcia Dias ${ }^{1}$ \\ Universidade Federal de Santa Maria, Santa Maria-RS, Brasil \\ Marco Antônio Pereira Teixeira \\ Universidade Federal do Rio Grande do Sul, Porto Alegre-RS, Brasil
}

\begin{abstract}
Resumo: O presente trabalho apresenta uma revisão seletiva e não sistemática da literatura a respeito do fenômeno da gestação na adolescência. Três eixos temáticos orientam a organização do trabalho: (a) Riscos e problemas associados à gestação na adolescência; (b) Fatores precursores relacionados à gravidez na adolescência; e (c) Fatores sócio-culturais associados ao desejo de ser mãe na adolescência. Entre as conclusões, destaca-se que a gravidez na adolescência é uma experiência que pode ter consequências tanto negativas quanto positivas para os adolescentes. Além disso, o fenômeno evidencia a necessidade de intervenções voltadas à saúde sexual e reprodutiva dos adolescentes.
\end{abstract}

Palavras-chave: gravidez na adolescência, adolescentes, sexualidade.

\section{Adolescent pregnancy: a look at a complex phenomenon}

\begin{abstract}
This article presents a selective and non-systematic literature review about the phenomenon of adolescent pregnancy. The text is organized along three themes: (a) risks and problems associated with adolescent pregnancy, (b) precursor factors related to pregnancy in adolescence, and (c) socio-cultural factors associated with the desire to become a mother in adolescence. Conclusions point out to the fact that adolescent pregnancy is an experience that can have both negative and positive consequences for adolescents. Additionally, the phenomenon highlights the need of interventions focused on adolescents' sexual and reproductive health.
\end{abstract}

Keywords: adolescent pregnancy, adolescents, sexuality.

\section{Embarazo en la adolescencia: una mirada a un fenomeno complejo}

\begin{abstract}
Resumen: Este artículo presenta una revisión selectiva y no sistemática de la literatura sobre el fenómeno del embarazo en la adolescencia. Tres temas guían la organización del texto: (a) los riesgos y los problemas asociados con el embarazo adolescente, (b) los factores precursores relacionados con el embarazo en la adolescencia y (c) los factores socioculturales asociados con el deseo de ser madre en la adolescencia. Entre las conclusiones, se destaca que el embarazo en la adolescencia es una experiencia que puede tener consecuencias tanto negativas cuanto positivas para los adolescentes. Por otra parte, el fenómeno pone de relieve la necesidad de intervenciones centradas en la salud sexual y reproductiva de los adolescentes.
\end{abstract}

Palabras clave: embarazo en adolescencia, adolescente, sexualidad.

Até aproximadamente meados do século XX, a gestação na adolescência não era considerada uma questão de saúde pública, e também não recebia a atenção de pesquisadores como recebe hoje em dia. No Brasil, esse fenômeno tornouse mais visível com o aumento da proporção de nascimentos em mães menores de 20 anos que se observou ao longo da década 90, quando os percentuais passaram de 16,38\% em 1991 para 21,34\% em 2000 (Instituto Brasileiro de Geografia e Estatística [IBGE], 2002). Deve-se considerar, no entanto, que parte desse aumento proporcional pode ser atribuído à diminuição expressiva das taxas de fecundidade nas faixas etárias acima de 25 anos. Estudos mais recentes sugerem que tanto a fecundidade adolescente quanto a proporção

1 Endereço para correspondência:

Prof ${ }^{\mathrm{a}}$. Dr ${ }^{\mathrm{a}}$. Ana Cristina Garcia Dias. Universidade Federal de Santa Maria. Centro de Ciências Sociais e Humanas. Departamento de Psicologia. Rua Floriano Peixoto, 1750. CEP 97.015-372. Santa MariaRS, Brasil. E-mail: cristcris@hotmail.com de nascimentos em mães adolescentes vêm diminuindo nos últimos anos (Yazaki, 2008).

Apesar de que, quantitativamente, o fenômeno possa até não estar aumentando (ou mesmo estar diminuindo), ele trouxe à tona algumas questões importantes: é a gravidez uma experiência esperada ou desejada na adolescência? O que ela revela? Quais suas consequências? O interesse pelo assunto é decorrência, em boa parte, do aumento das preocupações que tem havido em torno das questões que envolvem a adolescência, que não se define apenas a partir de critérios etários ou biológicos (Oliveira, 2008). Ela é, antes de tudo, um fenômeno social, um nome que se dá a um período do desenvolvimento no qual certas expectativas sociais recaem sobre os indivíduos e configuram um modo de ser adolescente, fruto da conjugação de transformações biológicas, cognitivas, emocionais e sociais pelas quais passam as pessoas. Sendo a gravidez um fenômeno social, os contornos da adolescência não podem ser definidos em termos absolutos, uma vez que tal definição depende do lugar que a sociedade atribui ao adolescente em um dado momento histórico (Teixeira \& Dias, 2004). 
Dessa forma, não apenas o conceito de adolescência pode mudar ao longo do tempo, como também podem coexistir diferentes modos de entender e viver essa fase da vida, dependendo dos contextos sociais específicos dentro dos quais cada indivíduo se desenvolve Nesse sentido, observase que, com o aumento da industrialização e da urbanização na sociedade ocidental moderna, esse período da vida entre a infância e a vida adulta passou a ser entendido como uma etapa de transição, onde a preparação para o trabalho (através da escolarização) e a construção de um senso pessoal de identidade seriam elementos centrais (Erikson, 1976).

Dentro dessa lógica, a gravidez na adolescência seria uma experiência indesejada, dado que restringiria as possibilidades de exploração de identidade e de preparação para o futuro profissional. Em função disso, a gravidez na adolescência passou a ser vista como uma situação de risco biopsicossocial, capaz de trazer consequências negativas não apenas para as adolescentes, mas para toda a sociedade. Tornou-se, por isso, um problema social e de saúde pública. De fato, atualmente, a literatura biomédica utiliza expressões como gravidez precoce, indesejada, não-planejada e de risco para descrever e enfatizar as consequências sociais e biológicas negativas associadas ao fenômeno (Dias \& Aquino, 2006; Gonçalves \& Knauth, 2006; Pantoja, 2003). Assim, estabeleceu-se uma idéia implícita de adolescência na qual a gestação não está incluída como experiência normativa. Pelo contrário, ela é vista como um desvio de percurso, um evento supostamente não desejado pelas adolescentes e cujas consequências frustram o que seria considerada uma "boa" adolescência (Oliveira, 2008). Todavia, é necessário também questionar até que ponto adolescência e gravidez são experiências que conflitam entre si, o que certamente depende do modo como se entende a própria adolescência - tanto em termos teóricos quanto em termos do que se espera socialmente de um adolescente.

Cabe nos perguntarmos qual é o espaço que vem sendo dado ao adolescente na sociedade contemporânea, e também nos questionarmos acerca do modo como entendemos e lidamos com a sua sexualidade e o tipo de atenção que damos à sua saúde e aos seus direitos sexuais e reprodutivos. Para compreender a gravidez na adolescência e suas consequências é necessário reconhecer que este é um fenômeno complexo e multideterminado, que está associado a fatores psicológicos, sociais e históricos.

O presente trabalho apresenta uma revisão seletiva e não sistemática da literatura a respeito do fenômeno da gestação na adolescência. Três eixos temáticos foram escolhidos a priori para orientar a organização do trabalho: (a) Riscos e problemas associados à gestação na adolescência; (b) Fatores precursores relacionados à gravidez na adolescência; e (c) Fatores sócio-culturais associados ao desejo de ser mãe na adolescência. Optou-se por uma revisão não sistemática da literatura porque o objetivo central do trabalho não foi apresentar o estado da arte sobre o assunto, e sim desenvolver uma reflexão sobre a problemática da gravidez na adolescência, tendo como parâmetro os eixos temáticos norteadores. Inicialmente, foi realizado um levantamento na base de dados SciELO Brasil, usando as palavras-chave "gravidez" e "adolescência" para o período 2000-2009, o que gerou a identificação de 129 artigos. A partir dos resumos, alguns destes artigos foram considerados mais relevantes para a composição da discussão de cada um dos eixos e foram selecionados para aprofundamento. Além disso, eventualmente outras referências bibliográficas indicadas nos artigos também foram consultadas, sendo inseridas no corpo desse trabalho. As seções seguintes apresentam uma apreciação crítico-reflexiva do material consultado, conforme os eixos temáticos escolhidos.

\section{Riscos e problemas associados à gestação na adolescência}

A gestação na adolescência é considerada uma situação de risco biológico tanto para as adolescentes como para os recém-nascidos. Alguns autores observam que características fisiológicas e psicológicas da adolescência fariam com que uma gestação nesse período se caracterizasse como uma gestação de risco. Há evidências de que gestantes adolescentes podem sofrer mais intercorrências médicas durante gravidez e mesmo após esse evento que gestantes de outras faixas etárias. Algumas complicações como tentativas de abortamento, anemia, desnutrição, sobrepeso, hipertensão, (pré)eclampsia, desproporção céfalo-pélvica, hipertensão e depressão pósparto estão associadas à experiência de gravidez na adolescência (Belarmino, Moura, Oliveira, \& Freitas, 2009; Freitas \& Botega, 2002; Furlan e cols., 2003; Michelazzo e cols., 2004; Silveira, Oliveira, \& Fernandes, 2004; Yzalle e cols., 2002). Além disso, a gestação em adolescentes pode estar relacionada a comportamentos de risco como, por exemplo, a utilização de álcool e drogas ou mesmo a precária realização de acompanhamento pré-natal durante a gravidez (Caputo \& Bordin, 2007; Chalem e cols., 2007; Gama, Szwarcwald, \& Leal, 2002; Kassar, Lima, Albuquerque, Barbieri, \& Gurgel, 2006; Mitsuhiro, Chalem, Barros, Guinsburg, \& Laranjeira, 2006; Sina, Valdivieso, \& Del Pino, 2003).

Por outro lado, no que tange à saúde do bebê, a gestação na adolescência encontra-se associada a situações de prematuridade, baixo peso ao nascer, morte perinatal, epilepsia, deficiência mental, transtornos do desenvolvimento, baixo quociente intelectual, cegueira, surdez, aborto natural, além de morte na infância (Aquino-Cunha, Queiroz-Andrade, Tavares-Neto, \& Andrade, 2002; Gama, Szwarcwald, Leal, \& Filha, 2001). O bebê prematuro apresenta maiores riscos na adaptação à vida extra-uterina devido à imaturidade dos órgãos e sistemas; além de uma maior vulnerabilidade ao desenvolvimento de doenças. Os riscos da gestação na adolescência ainda estão associados à baixa adesão ao atendimento pré-natal demonstrado pelas adolescentes (Carniel, Zanolli, Almeida, \& Morcillo, 2006; Minagawa e cols., 2006). Cabe ressaltar que o acompanhamento pré-natal tem efeito protetor 
sobre a saúde da gestante e do recém-nascido, uma vez que contribui para uma menor incidência de mortalidade materna, baixo peso ao nascer e mortalidade perinatal (Gama e cols., 2002).

Yazlle e cols. (2002) e Kassar e cols. (2006) consideram que a ocorrência de problemas de saúde tanto na jovem como na criança pode estar mais relacionada ao estado de pobreza do que à idade da jovem propriamente. Os autores observam que uma boa parcela da população de gestantes adolescentes encontra-se em condições sócio-econômicas precárias, o que por sua vez está associado a uma maior ausência de condições adequadas de higiene, habitação, alimentação e saúde.

Em termos sociais, a gravidez na adolescência pode estar associada com pobreza, evasão escolar, desemprego, ingresso precoce em um mercado de trabalho não-qualificado, separação conjugal, situações de violência e negligência, diminuição das oportunidades de mobilidade social, além de maus tratos infantis (Almeida, Aquino, \& Barros, 2006; Dias \& Aquino, 2006; Estela e cols., 2003; Fonseca \& Araújo, 2004; Carniel e cols, 2006; Freitas \& Botega, 2002; Gama e cols., 2002; Lima e cols., 2004; Yazlle e cols., 2002). Contudo, as relações causais estabelecidas entre evasão escolar e gravidez na adolescência são controversas (Castro, Abramovay, \& Silva, 2004).

Há evidências de que jovens que evadem da escola possuem mais chances de tornarem-se gestantes adolescentes (Sabroza, Leal, Souza Jr., \& Gama, 2004), sugerindo que a evasão precede a gestação. Por outro lado, outras pesquisas indicam que a gestação na adolescência seria uma das causas da evasão escolar (Estela e cols., 2003; Oliveira, 1998; Lima e cols., 2004; Loss \& Sapiro, 2005). Almeida, Aquino e Barros (2006) indicam que ambos os fatores - tanto a evasão anterior à gestação $(20,5 \%)$ quanto a evasão posterior (40\%) - estão associadas ao fenômeno de gestação na adolescência. Oliveira (1998) refere que o abandono da escola pode ser fruto do constrangimento, da pressão de professores, de diretores e da própria família, que julgam essa situação como vexatória. Contudo, Castro e cols. (2004) apontam que as jovens abandonam os estudos porque se torna efetivamente mais difícil prosseguir nos mesmos, pois as adolescentes, além de cuidarem dos bebês, muitas vezes ingressam no mercado de trabalho. Essas autoras não encontraram, em sua pesquisa, os preconceitos descritos por Oliveira (1998). No entanto, efetivamente, professores, pais e jovens consideraram que a gravidez, neste momento da vida, diminui as oportunidades da adolescente e dificulta ou mesmo impossibilita aproveitar as experiências que a juventude poderia lhe proporcionar (Oliveira, 2005; Castro e cols., 2004).

No entanto, a experiência de gestação na adolescência não é necessariamente um fator limitador das oportunidades de escolarização e da busca por um futuro melhor. Em um estudo realizado com adolescentes paraenses, Pantoja (2003) observou que a maternidade adolescente fortaleceu a permanência da jovem na escola, uma vez que a escolaridade esteve associada, na concepção dessas jovens, às noções de mobilidade social e ao projeto de "ser alguém na vida". Assim, permanecer na escola foi visto como uma oportunidade para oferecer uma vida melhor ao filho.

Em termos psicológicos, a gestação na adolescência está associada à noção de risco na medida em que implica na vivência simultânea de dois fenômenos importantes do desenvolvimento: o ser adolescente e o ser mãe (Levandowski, Piccinini, \& Lopes, 2008). Tipicamente, ao menos entre as camadas economicamente mais favorecidas da população, a adolescência é considerada um período da vida no qual os jovens deveriam, na medida do possível, explorar possibilidades antes de tomar decisões que exigem maior comprometimento, como escolher uma profissão, casar e ter filhos (Erikson, 1968/1976). Porém, a maternidade na adolescência traz consigo uma série de expectativas e responsabilidades que limitam essas possibilidades de exploração, ao mesmo tempo em que institui um novo espaço de constituição da identidade (Rangel \& Queiroz, 2008; Carvalho, Merighi, \& Jesus, 2009).

A adolescente que engravida, além de exercer o papel de filha, passa a exercer o papel de mãe, e ressignifica, nesse processo, a sua relação com a própria mãe (Andrade, Ribeiro, \& Silva, 2006; Daadorian, 2003). A posição da adolescente gestante, no contexto familiar, é redimensionada, na medida em que ela precisa desenvolver habilidades e assumir responsabilidades relacionadas ao cuidado do bebê e de si mesma. A família também passa a ter expectativas em relação ao seu desempenho como mãe e em relação ao seu futuro. Independente de ter ou não desejado ser mãe, o papel materno se impõe para a adolescente e passa a assumir um espaço significativo na sua vida (Falcão \& Salomão, 2005; Silva \& Salomão, 2003). A maternidade exige que a jovem redefina sua identidade levando em consideração o fato de que sua vida, da gravidez em diante, estará vinculada às demandas do filho. A projeção de si mesmo no futuro, elemento importante da construção da identidade na adolescência é substancialmente afetada no caso das adolescentes que engravidam, que precisam lidar com uma nova perspectiva temporal dada pelo desenrolar da gravidez e do próprio desenvolvimento do bebê após o nascimento. Planos são deixados de lado ou redimensionados em função da gestação e da maternidade/ paternidade (Fonseca \& Araújo, 2004).

Contudo, adaptar-se ao papel materno, ao mesmo tempo em que é adolescente e filha, não é uma tarefa fácil para a jovem. De fato, as transformações emocionais e cognitivas características pelas quais as adolescentes passam nesse período do desenvolvimento fazem com que as jovens apresentem mais dificuldades para desempenhar de maneira satisfatória o papel materno, uma vez que não dispõem, na maior parte das vezes, dos recursos psicológicos necessários para entender e tolerar as demandas diárias e frustrações da maternidade (Silva \& Salomão, 2003). Enfim, as dificuldades, inseguranças e falta de habilidades para o exercício do papel materno, associadas ao pouco conhecimento sobre desenvolvimento infantil que as adolescentes possuem, podem 
se configurar em um quadro de risco para o desenvolvimento do bebê, uma vez que as respostas das jovens mães às demandas de seus filhos tende a ser aquém ou além das suas necessidades (Bigras \& Paquette, 2007). Contudo alguns estudos mostram que, se a jovem recebe apoio, ela pode superar essas dificuldades (Andrade, Ribeiro, \& Silva, 2006; Silva, Nakano, Gomes, \& Stefanello, 2009).

\section{Fatores precursores relacionados à gravidez na adolescência}

O motivo óbvio e direto da gravidez na adolescência é o fato de que os adolescentes mantêm relações sexuais sem cuidados contraceptivos. Portanto, dois comportamentos precisam existir para que ocorra a gravidez na adolescência: a atividade sexual do jovem e a falta de medidas contraceptivas adequadas. Uma compreensão das causas desse fenômeno deve considerar a inter-relação entre esses comportamentos.

A iniciação sexual na adolescência vem ocorrendo em idades cada vez mais precoces, e a atividade sexual regular faz parte de uma parcela significativa da população adolescente (Cano, Ferriani, \& Gomes, 2000; Vieira, Saes, Dória, \& Goldberg, 2006). Essas mudanças no comportamento sexual são resultado de transformações nos valores que tiveram início nos anos 60 e trouxeram consequências importantes para $\mathrm{a}$ área da sexualidade humana. A literatura indica que novos padrões de comportamentos sexuais surgiram a partir do surgimento da pílula anticoncepcional. Este dispositivo contraceptivo, mais eficaz que os anteriormente utilizados, permitiu que o sexo, que estava intimamente vinculado à função reprodutiva, pudesse ter um descolamento da mesma e fosse focalizado sob a ótica do prazer (Cano, Ferriani, \& Gomes, 2000; Neiverth \& Alves, 2002). Essa desvinculação ocorreu de tal forma que hoje é difícil para o adolescente associar o sexo com a possibilidade de procriação e assim adotar um comportamento contraceptivo eficaz (Dias \& Gomes, 1999; Dias \& Gomes, 2000).

Além disso, essa "liberdade sexual" não foi necessariamente acompanhada por uma discussão de valores associados ao corpo, à sexualidade e aos papéis sexuais e de gênero presentes em nossa sociedade. Mensagens contraditórias são oferecidas constantemente aos jovens; por trás de uma aparente liberalidade ou indiferença, encontra-se, muitas vezes, uma moralidade rígida e punitiva, quando os valores familiares são transgredidos. Além disso, cabe ressaltar que os padrões sexuais impostos para meninos e meninas são diferentes (Amaral \& Fonseca, 2006; Cabral, 2003; Dias \& Gomes, 1999; Neiverth \& Alves, 2002).

Essa contradição nos valores é experienciada pelos adolescentes através do fenômeno que Takiuti (1989) denominou como "o querer versus o não poder". Por um lado, a família reprime a sexualidade do adolescente; por outro, o grupo de pares pressiona tanto meninos como meninas a expressarem e experimentarem suas sexualidades. Amaral e
Fonseca (2006) observaram que desejo e medo perpassam as representações dos adolescentes sobre sua iniciação sexual. Outros estudos mostram que a ausência de um comportamento contraceptivo em jovens se encontra associada à ambiguidade de valores sociais em relação ao corpo, à sexualidade e ao gênero transmitidos aos adolescentes (Alves \& Brandão, 2009; Cabral, 2003; Dias \& Gomes, 1999; Gonçalves \& Knauth, 2006; Pantoja, 2003).

Essa ambiguidade pode ser percebida tanto nas expectativas como nos comportamentos considerados apropriados para cada sexo. Gonçalves e Knauth (2006) lembram que se espera da mulher um comportamento passivo, enquanto do homem é esperado um comportamento ativo. Assim, o despreparo apresentado por muitas adolescentes em sua primeira relação confirmaria essa atitude passiva. Preparar-se para uma relação, que pode ser indicado através da adoção de um comportamento contraceptivo adequado, implica em uma postura ativa da mulher, que pode ser interpretada como experiência sexual ou "vontade de". Esses comportamentos considerados ativos colocariam em cheque a moralidade feminina. Assim, a vivência da sexualidade na mulher é considerada moralmente correta se ocorre de forma inocente, sem premeditação, movida pela paixão. Essa produção da "inocência" na jovem sexualmente ativa substitui o valor que a virgindade possuía em momentos anteriores em relação à regulação da sexualidade feminina (Desser, 1993). Tal atitude passiva, por parte das adolescentes, pode levar a relações sexuais desprotegidas e, por consequência, a gestações indesejadas. Por outro lado, os adolescentes homens não são educados para também se responsabilizarem pelos cuidados anticoncepcionais, deixando tais cuidados muitas vezes apenas para as meninas (Alves \& Brandão, 2009; Amaral \& Fonseca, 2006; Cabral, 2003; Heilborn e cols., 2002).

A causa do não uso de anticoncepcionais, portanto, não parece ser a falta de informação sobre a necessidade de se utilizar métodos contraceptivos nas relações sexuais. Algumas pesquisas mostram que, entre adolescentes que engravidaram, muitas sabiam que corriam o risco de gravidez e que poderiam ter usado algum contraceptivo (Dias \& Gomes, 2000; Guimarães \& Witter, 2007). O que ocorre é que a informação não se traduz em comportamento efetivo. E por que isso? Um motivo é que a informação que os adolescentes possuem refere-se à necessidade de uso de contraceptivos, mas não significa que eles possuam conhecimento suficiente para implementar um comportamento contraceptivo adequado. Há estudos mostrando que os conhecimentos sobre métodos de contracepção entre adolescentes são muitas vezes insuficientes para uma efetiva implementação (Gomes, Costa, Sobrinho, Santos, \& Bacelar, 2002; Silva, Bomfim, Cardozo, Franco, \& Marques, 2007; Sousa \& Gomes, 2009).

Por exemplo, Belo e Silva (2004) observaram, em um estudo com gestantes adolescentes do município de Campinas, que $67,3 \%$ das jovens, apesar de possuírem um bom nível de conhecimento sobre métodos contraceptivos, não 
utilizaram método algum na primeira relação. As principais razões citadas para o não uso dos métodos contraceptivos foram: não pensaram nisso na hora $(32,4 \%)$; desejavam a gravidez $(25,4 \%)$; não esperavam ter relação sexual naquele momento $(12,7 \%)$; não conheciam nenhum método contraceptivo $(11,3 \%)$, os parceiros não queriam usar $(8,5 \%)$, não se importavam em ficar grávidas $(5,6 \%)$, achavam caro ou inconveniente usar algum contraceptivo $(5,6 \%)$. Dados como esses mostram que, mesmo quando existe conhecimento suficiente e acesso a algum método contraceptivo, pode existir ambivalência quanto ao uso, pois utilizá-lo implica assumir e expressar a sua sexualidade, o que pode ser algo difícil para os adolescentes, especialmente as mulheres, como já apontado anteriormente. Além disso, outros fatores de ordem cognitiva e afetiva também podem estar presentes.

Do ponto de vista cognitivo, sabe-se que os adolescentes, particularmente os mais jovens, têm dificuldade em avaliar a extensão e o impacto das consequências do próprio comportamento. Os adolescentes podem se sentir invulneráveis, não acreditando que a gravidez possa acontecer consigo, apesar de ocorrer com outros jovens (Loss \& Sapiro, 2005; Santos \& Carvalho, 2006; Ximenes Neto, Dias, Rocha, \& Cunha, 2007). Ou então podem considerar que, como nenhum de seus amigos adolescentes já engravidou, então isso também não aconterá com eles (Vilella \& Doreto, 2006). Essas crenças estão associadas a não adoção de um comportamento contraceptivo adequado. De fato, a capacidade cognitiva de avaliar consequências adequadamente e de trabalhar com hipóteses pode não estar bem estabelecida na adolescência (Inhelder \& Piaget, 1976).

Já em termos afetivos, a gestação adolescente pode ser associada a características da própria adolescência como: dificuldades no controle dos impulsos, na separação dos pais e na constituição da própria identidade (Dadorian, 2003; Santos \& Carvalho, 2006). A gravidez na adolescência, nesse sentido, poder estar relacionada a uma crise de dessimbiotização, ou seja, a dificuldades na elaboração da ruptura do vínculo de dependência simbiótica. A gestante buscaria solucionar esse conflito através da recriação da situação simbiótica (mãe-bebê), na qual pode se manter em um vínculo simbiótico com o filho. Ou seja, de acordo com esta perspectiva, a gravidez na adolescência seria resultado de um processo inconsciente no qual a jovem, impossibilitada de assumir sua autonomia emocional e de identidade por não conseguir separar-se psicologicamente da mãe, tentaria manter-se em um estado emocional fusional, transferindo esta dependência de vínculo à figura do filho ou filha (Deutsch, 1974).

Deutsch (1974) considera que uma quebra precoce na relação de apego da filha com a mãe geraria, além de um sentimento desesperado de solidão, um intenso desejo de união; a jovem buscaria reviver o vínculo mãe-filha através da maternidade. Para a autora, a gestação na adolescência seria um "ato compulsivo", no qual seriam reforçados os laços de dependência. Cabe lembrar que, muitas vezes, esses laços de identificação e vinculação podem ser intensificados.
Isso pode ocorrer tanto na situação em que a própria mãe da adolescente foi gestante adolescente como naquela na qual a jovem "doa" seu filho para a mãe criá-lo, reservando para si o papel de irmã mais velha.

\section{Fatores sócio-culturais associados ao desejo de ser mãe na adolescência}

Alguns estudos demonstram que a gestação na adolescência pode ser desejada e considerada uma experiência gratificante, apesar dos inúmeros problemas descritos na literatura sobre o tema (Levandowski, Piccinini, \& Lopes, 2008). Algumas pesquisas mostram que a gravidez nesse período pode representar a busca por reconhecimento e concretização de um projeto de vida viável para algumas adolescentes, especialmente aquelas de nível sócio-economico menos favorecido (Belo \& Silva, 2004; Carvalho, Merighi, \& Jesus, 2009; Dadoorian, 2003; Oliveira, 2005; Pantoja, 2003; Rangel \& Queiroz, 2008).

Além disso, Reis e Oliveira-Monteiro (2007) observaram que a falta de oportunidades de vida e as carências emocionais se encontram associadas à maternidade na adolescência e ao desejo de ter um filho. Em um estudo com jovens participantes de um programa de inclusão sócio-cultural, com moradores de uma favela na grande São Paulo, os autores encontraram que, entre as meninas estudadas, "sentir-se só" (24\%), "brigas ou tristezas com a família" (23\%), "falta de opções na vida" (13\%) e "gostar de crianças" (10\%) foram as principais razões indicadas para o desenvolvimento de uma gravidez na adolescência. Já entre os meninos, a gestação foi relacionada principalmente com "falta de opções na vida" (25\%), "brigas ou tristezas com a família" (15\%), "vontade de ter a própria família" (15\%), "gostar de crianças" (10\%) e a "falta de oportunidades de estudar ou trabalhar" (10\%). Nesse sentido, conclui-se que a maternidade na adolescência foi compreendida pelos jovens como uma alternativa viável para lidar com uma série de problemas e situações desfavoráveis presentes em seu contexto sócio-afetivo. Desta forma, a carência afetiva associada à ausência ou limitação nas perspectivas de construção de um projeto de vida podem ser fatores determinantes para a ocorrência de uma gestação na adolescência, ao menos, em classes desprivilegiadas.

Assim, a gestação na adolescência se apresenta como um projeto viável e valorizado, em um contexto em que não existem muitas alternativas possíveis de implementação de outros projetos de vida. Alguns estudos (Carvalho, Merighi, \& Jesus, 2009; Desser, 1993; Loss \& Sapiro, 2005; Oliveira, 2005; Rangel \& Queiroz, 2008; Santos \& Carvalho, 2006; Ximenes Neto e cols., 2007) demonstram que a maternidade, para uma grande parcela das jovens, é desejada e se configura como um dos únicos projetos possíveis de reconhecimento social. Além de representar, em alguns casos, um modo de conceber a concretização da identidade feminina.

Gontijo e Medeiros (2008), por exemplo, encontraram que a maternidade esteve associada a sentimentos de 
satisfação pessoal e possibilitou às adolescentes com experiência de vida nas ruas uma oportunidade de realização pessoal. O filho foi percebido como uma pessoa que iria acabar com a sua solidão e sentimento de abandono, pois a jovem poderia de forma genuína dar amor a este filho e dele receber amor. Ele representaria um "salvador" da morte certa que encontrariam se continuassem na vivência de sua experiência de rua.

Rangel e Queiroz (2008), por sua vez, ao compararem as representações sociais de adolescentes de diferentes estratos econômicos sobre a gravidez nesse período do desenvolvimento, encontraram entre meninas de um nível econômico menos favorecido que ter um filho era uma bênção divina, algo "natural" da identidade feminina. Em suas representações, a maternidade estava vinculada ao "poder de ser mulher" e à construção da própria família. As autoras observaram que a representação da gravidez na adolescência esteve atrelada à necessidade de valorização e de um reconhecimento social, o que não ocorria com adolescentes dos estratos médios mais favorecidos. Entre essas jovens a gestação nesse momento de vida representava, além de um comprometimento dos planos futuros, uma sobrecarga financeira e uma experiência não normativa no desenvolvimento humano ("não era a hora").

Loss e Sapiro (2005) encontraram dados similares aos descritos anteriormente, observando que o "engravidamento" na adolescência representa tanto impasses como possibilidades entre jovens da periferia de Porto Alegre. Essas autoras utilizam o termo engravidamento para referir-se ao processo e às expectativas que jovens da periferia apresentam frente à experiência da gestação na adolescência. O engravidamento, entendido como a expectativa de estar grávida, ressalta tanto o poder constitutivo da maternidade na construção da identidade feminina como a importância dessa experiência compartilhada nesse contexto sócio-cultural, no qual o papel materno é extremamente valorizado. As autoras destacam que a maternidade adolescente apresenta uma dimensão coletiva e intergeracional, compartilhada entre os membros da comunidade na qual a adolescente encontra-se inserida.

Esse aspecto de compartilhamento de valores e inserção social da gestação adolescente também é apontado por Pantoja (2003). Esse autor revelou, em um estudo socio-antropológico realizado com adolescentes de Belém do Pará, que a gravidez na adolescência pode ser valorizada pelas jovens, pois traduz uma mudança em seu status social. A autora observou que, embora algumas meninas realizem abortos ou até procurem ocultar a gestação, o que confirmaria a visão tradicional da gravidez como um problema de saúde pública, há diferentes práticas e discursos que indicam o significado positivo desta experiência para as adolescentes e para outras pessoas do contexto sócio-cultural próximo. Ela encontrou, por exemplo, que na escola é construída uma rede de relações entre as adolescentes grávidas e aquelas que já vivenciaram essa experiência, sendo compartilhadas experiências e conselhos em como lidar com essa nova situação.
De fato, a autora constatou que a gravidez/maternidade envolve dimensões complexas, pois neste contexto estudado, além de uma mudança de status social da adolescente frente ao grupo, pôde-se perceber a reafirmação de projetos de mobilidade social pela adolescente a partir de sua gravidez. A gravidez/maternidade na adolescência pode fazer parte do projeto de vida das adolescentes, uma vez que funciona como uma espécie de "passaporte" para entrar na vida "adulta". Esse fenômeno parece demarcar, neste contexto estudado, a entrada da jovem no mundo adulto, de maneira legítima, uma vez que a adolescente passa a ser reconhecida como adulta pela família, professores e colegas de escola. Além disso, a gravidez/maternidade propicia às jovens a reafirmação de projetos de ascensão social, pois a busca por continuidade dos estudos, que demanda esforços tanto da jovem como de sua família, indica a procura por melhores condições de vida para si e para a futura criança. Assim, neste contexto, a opção pela maternidade e continuidade dos estudos significa manter-se firme no projeto de "ser alguém na vida" (Pantoja, 2003).

\section{Considerações finais}

A gravidez na adolescência é uma realidade que nos convoca a refletir sobre o assunto para buscar compreendêlo e, a partir desta compreensão, propor modos de lidar com o fenômeno. A revisão aqui apresentada mostrou que as consequências de uma gestação na adolescência tendem a ser negativas quando se olha a questão desde uma perspectiva estritamente biológica, ou então tomando-se como parâmetro as expectativas sociais do que seria um desenvolvimento típico na adolescência. Sem dúvida, existem evidências a indicar que há uma série de riscos para a saúde relacionados com a gravidez na adolescência, tanto para a mãe quanto para o bebê. Sabe-se, também, que as demandas da gestação e da maternidade implicam diversas transformações no modo de vida das adolescentes, o que acaba limitando ou prejudicando o seu envolvimento em atividades importantes para o seu desenvolvimento durante esse período da vida, como escola e lazer.

Conforme ressaltado pela literatura, porém, a gravidez na adolescência não é um fenômeno homogêneo. Dependendo do contexto social em que a adolescente vive, o significado da gestação, assim como o impacto dessa experiência de vida no desenvolvimento da jovem, pode assumir diferentes contornos. Em camadas sociais mais abastadas, por exemplo, a gravidez na adolescência tende a não prejudicar tanto o percurso de escolarização e profissionalização das jovens quanto nas camadas menos favorecidas, em virtude da maior disponibilidade de recursos e apoios para lidar com essa situação e suas demandas. Dessa forma, a perspectiva de futuro das adolescentes grávidas de classe média não é afetada tão intensamente quanto a perspectiva das adolescentes de classe baixa, considerando-se os aspectos de escolarização e profissionalização. 
Contudo, a análise do fenômeno da gravidez na adolescência não pode se resumir aos impactos negativos quanto às perspectivas de vida. As pesquisas mostram que, muitas vezes, a gravidez pode ser desejada pelas jovens, pois é tida como uma via de acesso a um novo estatuto de identidade e de reconhecimento através do papel materno. A maternidade, nesses casos, pode ser vista como uma ocupação, um papel que dá um sentido à vida da jovem. Na falta de outros projetos de vida, ou frente à dificuldade em vislumbrar a possibilidade de efetivar planos alternativos, a gravidez pode ser percebida pela adolescente como uma forma de reconhecer a si mesma, de marcar seu próprio espaço na família e de ser reconhecida nos seus ambientes de convívio.

Portanto, é preciso cuidado ao se emitir juízos de valor a respeito da gravidez na adolescência. Ainda que, do ponto de vista da saúde pública, esse seja um fenômeno com repercussões negativas, na medida em que implica riscos de saúde para mães e bebês, riscos de cuidados inadequados aos bebês e riscos de empobrecimento nas perspectivas de escolarização, trabalho e renda das adolescentes e suas famílias, ele também pode ter consequências consideradas positivas pelas adolescentes. Além disso, é preciso reconhecer que, nas camadas sociais nas quais as possibilidades educacionais e de ascensão social são restritas, a maternidade precoce pode ser um dos projetos de vida mais acessíveis para as adolescentes, devido à falta de outras oportunidades oferecidas em seu contexto de vida. Assim, pensar a gravidez na adolescência como algo não desejado ou fora do esperado corresponde a uma perspectiva normativa da adolescência que exclui a maternidade precoce como uma alternativa de vida. As evidências sugerem, contudo, que podem existir - e certamente existem - outros modos de ser adolescente e viver a adolescência, sendo que a maternidade pode fazer parte desses diferentes modos de ser adolescente, ainda que isso possa trazer consequências negativas, dependendo do ângulo sob o qual se analisa a questão.

Outro ponto que precisa ser considerado diz respeito ao tema da sexualidade, que está estreitamente vinculado à problemática da gravidez na adolescência. Focalizar a questão apenas na gestação e suas consequências é perder de vista o contexto dentro do qual a gravidez se produz. Intervenções que visem prevenir a gravidez na adolescência não devem se restringir a oferecer informações sobre métodos contraceptivos. Mais do que isso, elas devem buscar trabalhar, junto com os adolescentes, os significados e as ansiedades que estão envolvidos nos diversos comportamentos de paquera, iniciação sexual e de vida sexual ativa, de modo que as práticas contraceptivas passem a ser percebidas cada vez mais como algo positivo e natural, assim como a vivência da própria sexualidade. Da mesma forma, os significados e possíveis consequências de uma gravidez e da maternidade também precisam ser discutidos, incluindo aí o papel dos adolescentes (homens) na gestação e na paternidade. Entender o adolescente como um sujeito com direitos, tanto sexuais quanto reprodutivos, talvez seja o primeiro passo necessário para que ele possa reconhecer-se também como um sujeito que tem deveres em relação a sua própria sexualidade e, mais do que isso, que precisa ter responsabilidade para com a própria vida, em todos os seus aspectos.

\section{Referências}

Almeida, M. C. C., Aquino, E. M. L., \& Barros, P. (2006). School trajectory and teenage pregnancy in three Brazilian state capitals. Cadernos de Saúde Pública, 22, 1397-1409.

Alves, C. A., \& Brandão, E. R. (2009). Vulnerabilidade no uso de métodos contraceptivos entre adolescentes e jovens: intersecção de políticas públicas e atenção à saúde. Ciência e Saúde Coletiva, 14, 661-670.

Amaral, M. A., \& Fonseca, R. M. G. S. (2006). Entre o desejo e o medo: As representações sociais das adolescentes acerca da iniciação sexual. Revista Escola de Enfermagem - USP, 40, 469-476.

Andrade, P. R., Ribeiro, C. A., \& Silva, C. V. (2006). Mãe adolescente vivenciando o cuidado do filho: Um modelo teórico. Revista Brasileira de Enfermagem, 59, 30-35.

Aquino-Cunha, M., Queiroz-Andrade, M., Tavares-Neto, J., \& Andrade, T. (2002). Gestação na adolescência: Relação com baixo peso ao nascer. Revista Brasileira de Ginecologia e Obstetrícia, 24, 513-518.

Belarmino, G. O., Moura, E. R. F., Oliveira, N. C., \& Freitas, G. L. (2009). Risco nutricional entre gestantes adolescentes. Acta Paulista de Enfermagem, 22, 169-175.

Belo, M. A. V., \& Silva, J. L. P. (2004). Conhecimento, atitude e prática sobre métodos anticoncepcionais entre adolescentes gestantes. Revista Brasileira de Saúde Pública, 38, 479-487.

Bigras, M., \& Paquette, D. (2007). Estudo pessoa-processocontexto da qualidade das interações entre mãeadolescente e seu bebê. Ciência e Saúde Coletiva, 12, 1167-1174.

Cabral, C. S. (2003). Contracepção e gravidez na adolescência na perspectiva de jovens pais de uma comunidade favelada do Rio de Janeiro. Cadernos de Saúde Pública, 19(supl.2), s283-s292.

Cano, M. A. T., Ferriani, M. G. C., \& Gomes, R. (2000). Sexualidade na adolescência: Um estudo bibliográfico. Revista Latino-Americana de Enfermagem, 8(2), 18-24.

Caputo, V. G., \& Bordin, I. A. (2007). Problemas de saúde mental entre jovens grávidas e não grávidas. Revista de Saúde Pública, 41, 573-581.

Carniel, E. F., Zanolli, M. L., Almeida, C. A. A., \& Morcillo A. M. (2006). Características das mães adolescentes e de seus recém-nascidos e fatores de risco para a gravidez na adolescência em Campinas, SP, Brasil. Revista Brasileira de Saúde Materno-Infantil, 6, 419-426.

Carvalho, G. M., Merighi, M. A. B., \& Jesus, M. C. P. (2009). Recorrência da parentalidade na adolescência na perspectiva dos sujeitos envolvidos. Texto e Contexto Enfermagem, 18, 17-24. 
Castro, M. G., Abramovay, M., \& Silva, L. B. (2004). Juventudes e sexualidades. Brasília: UNESCO Brasil.

Chalem, E., Mitsuhiro, S. S., Ferri, C. P., Barros, M. C. M., Guinsburg, R., \& Laranjeira, R. (2007). Gravidez na adolescência: Perfil sócio-demográfico e comportamental de uma população da periferia de São Paulo, Brasil. Cadernos de Saúde Pública, 23, 177-186.

Dadoorian, D. (2003). Gravidez na adolescência: Um novo olhar. Psicologia Ciência e Profissão, 23(1), 84-91.

Desser, N. A. (1993). Adolescência, sexualidade e culpa. Brasília: Edunb.

Dias, A. B., \& Aquino, E. M. L. (2006). Maternidade e paternidade na adolescência: Algumas constatações em três cidades do Brasil. Cadernos de Saúde Pública, 22, 1447-1458.

Dias, A. C. G., \& Gomes, W. B. (2000). Conversas, em família, sobre sexualidade e gravidez na adolescência: Percepção de jovens gestantes. Psicologia: Reflexão e Crítica, 13, 109-125.

Dias, A. C. G., \& Gomes, W. B. (1999). Conversas sobre sexualidade na família e gravidez na adolescência: A percepção dos pais. Estudos de Psicologia (Natal), 4, 79106.

Deutsch, H. (1974). Problemas psicológicos da adolescência (E. Jorge, Trad.). Rio de Janeiro: Zahar. (Original publicado em 1967)

Erikson, E. H. (1976). Identidade, juventude e crise. (A. Cabral, Trad.). Rio de Janeiro: Zahar. (Original publicado em 1968)

Estela, M. L., Aquino, E. M., Heilborn, M. L., Knauth, D., Michel Bozon, M., Almeida, M. C., Araújo, J., \& Menezes, G. (2003). Adolescência e reprodução no Brasil: A heterogeneidade dos perfis sociais. Cadernos de Saúde Pública, 19(supl.2), s377-s388.

Falcão, D. V., \& Salomão, N. M. R. (2005). O papel dos avós na maternidade adolescente. Estudos de Psicologia (Campinas), 22, 205-212.

Fonseca, A. L. B., \& Araújo, N. G. (2004). Maternidade precoce: Uma das consequências do abandono escolar e do desemprego. Revista Brasileira de Crescimento e Desenvolvimento Humano, 14(2), 16-22.

Freitas, G. V. S., \& Botega, N. J. (2002). Gravidez na adolescência: Prevalência de depressão, ansiedade e ideação suicida. Revista da Associação Médica Brasileira, 48, 245-249.

Furlan, J. P., Guazelli, C. A. F., Papa, A. C. S., Quintino, M. P., Soares, R.V. P., \& Mattar, R. (2003). A influência do estado nutricional da adolescente grávida sobre o tipo de parto e o peso do recém-nascido. Revista Brasileira de Ginecologia e Obstetrícia, 25, 625-630.

Gama, S. G. N., Szwarcwald, C. L., \& Leal, M. C. (2002). Experiência de gravidez na adolescência, fatores associados e resultados perinatais entre puérperas de baixa renda. Cadernos de Saúde Pública, 18, 153-161.
Gama, S. G. N., Szwarcwald, C. L., Leal, M. C., \& Filha, M. M. T. (2001). Gravidez na adolescência como fator de risco para baixo peso ao nascer no município do Rio de Janeiro, de 1996 a 1998. Revista de Saúde Pública, $35,74-80$.

Gomes, W. A., Costa, M. C., Sobrinho, C. L. N., Santos, C. A. S. T., \& Bacelar, E. B. (2002). Nível de Informação sobre adolescência, puberdade e sexualidade entre adolescentes. Jornal de Pediatria, 78, 301-308.

Gonçalves, H., \& Knauth, D. R. (2006). Aproveitar a vida, juventude e gravidez. Revista de Antropologia, 49, 625-643.

Gontijo, D. T., \& Medeiros, M. (2008). "Tava morta e revivi": Significado de maternidade para adolescentes com experiência de vida nas ruas. Cadernos de Saúde Pública, 24, 469-472.

Guimarães, E. A., \& Witter, G. P. (2007). Gravidez na adolescência: Conhecimentos e prevenção entre jovens. Boletim Academia Paulista de Psicologia, 27(2), 167180.

Heilborn, M. L., Salem, T., Rohden, F., Brandão, E., Knauth, D., Victoria, C., Aquino, E., McCallun, C., \& Bozon, M. (2002). Aproximações socioantropológicas sobre a gravidez na adolescência. Horizontes Antropológicos, $8(17), 13-45$.

Instituto Brasileiro de Geografia e Estatística. (2002). Estatística do Registro Civil (vol. 29). Rio de Janeiro: IBGE.

Inhelder, B., \& Piaget, J. (1976). O pensamento adolescente (D. M. Leite, Trad). In J. Piaget \& B. Inhelder (Orgs.), Da lógica da criança à lógica do adolescente: Ensaios sobre a construção das estruturas operatórias formais (pp. 249-259). São Paulo: Pioneira. (Original publicado em 1955)

Kassar, S. B., Lima, M. C., Albuquerque, M. F. M., Barbieri, M. A., \& Gurgel, R. Q. (2006). Comparação de condições socioeconômicas e reprodutivas entre mães adolescentes e adultas jovens em três maternidades públicas de Maceió, Brasil. Revista Brasileira de Saúde Materno-Infantil, 6, 397-403.

Levandowski, D. C., Piccinini, C. A., \& Lopes, R. C. S. (2008). Maternidade adolescente. Estudos de Psicologia (Campinas), 25, 251-263.

Lima, C. T. B., Feliciano, K. V. de O., Carvalho, M. F. S., Souza, A. P. P., Menabó, J. B. C., Ramos, L. S., Cassundé, L. F., \& Kovács, M. H. (2004). Percepções e práticas de adolescentes grávidas e de familiares em relação à gestação. Revista Brasileira de Saúde Materno-Infantil, 4, 71-83.

Loss, M. A., \& Sapiro, C. M. (2005). Processos psíquicos do engravidamento na adolescência em contexto de periferia: Impasses e possibilidades. Psicologia USP, 16(4), 69-98. 
Michelazzo, D., Yazlle, M. E. H. D., Mendes, M. C., Patta, M. C., Rocha, J. S. Y., \& Moura, M. D. (2004). Indicadores sociais de grávidas adolescentes: Estudo de caso-controle. Revista Brasileira de Ginecologia e Obstetrícia, 26, 633-639.

Minagawa, A. T., Biagoline, R. E. M., Fujimori, E., Oliveira, I. M. V., Moreira, A. P. C. A., \& Ortega, L. D. S. (2006). Baixo peso ao nascer e condições maternas no pré-natal. Revista da Escola de Enfermagem da USP, 40, 548-554.

Mitsuhiro, S. S., Chalem, E., Barros, M. M., Guinsburg, R., \& Laranjeira, R. (2006). Teenage pregnancy: Use of drugs in the third trimester and prevalence of psychiatric disorders. Revista Brasileira de Psiquiatria, 28, 122-125.

Neiverth, I. S., \& Alves, G. B. (2002). Gravidez na adolescência e mudança no papel social da mulher. Paidéia (Ribeirão Preto), 12, 229-240.

Oliveira, M. W. (1998). Gravidez na adolescência: Dimensões do problema. Cadernos da CEDES, 19(45), 48-70.

Oliveira, N. R. (2005). Maternidade de adolescentes de periferias sociais e urbanas: Algumas análises à luz da Psicologia Ambiental. Revista Brasileira de Crescimento e Desenvolvimento Humano, 15(1), 69-77.

Oliveira, R. C. (2008). Adolescência, gravidez e maternidade: A percepção de si e a relação com o trabalho. Saúde $e$ Sociedade, 17(4), 93-102.

Pantoja, A. L. N. (2003). "Ser alguém na vida": Uma análise sócio-antropológica da gravidez/maternidade na adolescência, em Belém do Pará, Brasil. Cadernos de Saúde Pública, 19 (sup.2), s335-s343.

Rangel, D. L. O., \& Queiroz, A. B. A. (2008). Arepresentação social das adolescentes sobre a gravidez nessa etapa da vida. Escola Anna Nery Revista de Enfermagem, 12, 780-788.

Reis, A. O. A., \& Oliveira-Monteiro, N. R. (2007). Sexualidade e procriação na ótica de jovens de periferias sócias e urbanas. Revista Brasileira de Crescimento e Desenvolvimento Humano, 17(2), 54-63.

Santos, A., \& Carvalho, C. V. (2006). Gravidez na adolescência: Um estudo exploratório. Boletim de Psicologia, 56, 135-151.

Sabroza, A. R., Leal, M. C., Souza Jr., P. R., \& Gama, S. G. N. (2004). Algumas repercussões emocionais negativas da gravidez precoce em adolescentes do município do Rio de Janeiro (1999-2001). Cadernos de Saúde Pública, 20(sup.1), s130-s137.

Sina, D. E., Valdivieso, J. B., \& Del Pino, L. V. (2003). Natalidad y riesgo reproductivo en adolescentes de Chile, 1990-1999. Revista Panamericana de Salud Publica, 14(1), 3-8.

Silva, D. V., \& Salomão, N. M. R. (2003). A maternidade na perspectiva de mães de adolescentes e avós maternas de bebês. Estudos de Psicologia (Campinas), 8(1), 135-145.
Silva, L. A., Nakano, A. M. S., Gomes, F. A., \& Stefanello, J. (2009). Significados atribuídos por puérperas adolescentes à maternidade: Autocuidado e cuidado com o bebê. Texto e Contexto em Enfermagem, 18, 48-56.

Silva, N. C. B., Bomfim, T., Cardozo, N. P., Franco, M. A. P., \& Marques, S. L. (2007). Proposta de instrumento para avaliar conhecimento sobre métodos contraceptivos. Paidéia (Ribeirão Preto), 17, 365-374.

Silveira, I. P., Oliveira, M. I. V., \& Fernandes, A. F. C. (2004). Perfil obstétrico de adolescentes de uma maternidade pública do Ceará. Escola Anna Nery - Revista de Enfermagem, 8, 205-210.

Sousa, M. C. R., \& Gomes, K. R. O. (2009). Conhecimento objetivo e percebido sobre contraceptivos hormonais orais entre adolescentes com antecedentes gestacionais. Cadernos de Saúde Pública, 25, 645-654.

Takiuti, A. (1989). A adolescente está ligeiramente grávida. E agora? São Paulo: Iglu.

Teixeira, M. A. P., \& Dias, A. C. G. (2004). Quando termina a adolescência? Perspectiva (Erexim), 28, 7-15.

Vieira, L. M., Saes, S. O., Dória, A. A. B., \& Goldberg, T. B. L. (2006). Reflexões sobre a anticoncepção na adolescência no Brasil. Revista Brasileira de Saúde Materno-Infantil, 6, 135-140.

Villela, W. V., \& Doreto, D. T. (2006). Sobre a experiência sexual dos jovens. Cadernos de Saúde Pública, 22, $2467-$ 2472.

Ximenes Neto, F. R. G., Dias, M. S. A., Rocha, J., \& Cunha, I. C. K. O. (2007). Gravidez na adolescência: Motivos e percepções das adolescentes. Revista Brasileira de Enfermagem, 60, 279-285.

Yazlle, M. E. H. D., Mendes, M. C., Patta, M. C., Rocha, J. S. Y., Azevedo, G. D., \& Marcolin, A. C. (2002). A adolescente grávida: Alguns indicadores sociais. Revista Brasileira de Ginecologia e Obstetrícia, 24, 609-614.

Yazaki, L. M. (2008). Maternidades sucessivas em adolescentes no Estado de São Paulo. Anais do Encontro Nacional de Estudos Populacionais. Recuperado em 20 junho 2009, de www.abep.nepo.unicamp.br/ encontro2008/docsPDF/ABEP2008_1170.pdf

Ana Cristina Garcia Dias é Professora Adjunta do Departamento de Psicologia da Universidade Federal de Santa Maria.

Marco Antônio Pereira Teixeira é Professor Adjunto do Instituto de Psicologia da Universidade Federal do Rio Grande do Sul. 